$44,7,55:$ In omnibus rebus, quae dominium transferunt, concurrat oportet affectus ex utraque parte contrahentium: nam siue ea uenditio siue donatio siue dotis datio ${ }^{1}$ ) siue quaelibet alia causa contrahendi fuit, nisi animus utriusque consentit, perduci ad effectum id quod inchoatur non potest.

44, 7, 56: Quaecumque actiones serui mei nomine mihi coeperunt competere uel ex duodecim tabulis, ue lut $i^{2}$ ) iniuriarum uel furti, uel ex lege Aquilia ${ }^{3}$ ), eaedem durant, etiamsi seruus postea uel manumissus uel alienatus uel mortuus fuerit. . . .

$44,7,61,1: \ldots$ quaero, cum et rem suam distraxerit Lucius Titius et ad $S e i\left(\mathrm{~m}^{4}\right)$ profectus sit et ex eo cum ea sit, an ei ex his epistulis salarium annuum debeatur. . . .

47, 7, 5: Caedere est non solum succidere, sed etiam ferire caedendi causa. cingere est deglabrare. subsecare est subserrare ${ }^{5}$ ): non enim poterat cecidisse intellegi, qui serra secuisset.

\title{
XIII. \\ Ueber das \\ Halberstädter Bruchstück einer Handschrift des Codex Theodosianus.
}

\author{
Von \\ Herrn Professor W. Schum \\ in Halle.
}

Bei der Catalogisirung der Handschriftensammlung des Kgl. Dom-Gymnasiums zu Halberstadt, die Ende der siebziger Jahre in Verfolg einer höheren Ortes gegebenen allgemeinen Anregung bewirkt wurde, fand der Director der genannten Anstalt, Herr Dr. G. Schmidt, auf dem Umschlage

1) conductio $F M .-{ }^{2}$ ) nel $\left.F M .-{ }^{3}\right)$ hic ins. $F M$ : uel iniuriarum uel furti. - ${ }^{4}$ ) eam $\boldsymbol{F} M$. - 5) subsecuisse $F M$. 
des Manuscriptes $\mathrm{Nr} .22$, eines dem 14. Jahrhundert angehörenden Bandes mit Auszügen aus medicinischen und naturwissenschaftlichen Werken, zwei Pergamentblätter mit sehr alter Schrift und farbigen Bildern, die er sofort mit gutem Erfolge ablöste und mir zu näherer Prüfung freundlíchst überliess; auf Grund einer vorläufigen Untersuchung derselben durch mich gab Herr Director Dr. Schmidt hierauf im Osterprogramm des Dom-Gymnasiums von 1881, S. 22 und 23, eine weitere Nachricht von dem Funde; ich selbst liess sodann, da wir es mit Palimpsesten zu thun hatten und ich bei Anwendung von Reagentien zur Lesbarmachung des älteren Textes eine Zerstörung oder Beschädigung der jüngeren Schrift fürchtete, im Jahre 1881, so gut es gehen wollte, die Blätter photographiren und sandte Abzüge von diesen Aufnahmen, mit Bemerkungen begleitet, an Herrn Geh. Reg.-Rath Prof. Wattenbach ein, worauf derselbe im Neuen Archive der Gesellschaft, für ältere deutsche Geschichtskunde VII, 244 eine kurze Notiz von dem Sachverhalte brachte. Durch andere Arbeiten in Anspruch genommen, konnte ich eine ganze Reihe von Jahren hindurch den in Rede stehenden Blättern keine weitere Aufmerksamkeit widmen, als dass ich Herrn Professor Dr. R. Peiper in Breslau auf seinen Wunsch eine genaue Abschrift des oberen Textes fertigte; doch hatte Herr Director Schmidt die Güte, mir den Schatz, dessen ich mich gern zur Vorlage bei meinen palaeographischen Vorlesungen und Uebungen bediente, bisher zu belassen; es drängt mich nunmehr, denselben zurückzuliefern, doch möchte ich ihn nicht aus der Hand geben, ohne die für den älteren Text der Blätter sich gewiss interessirenden juristischen Kreise auf dieselben hierdurch noch einmal aufmerksam gemacht zu haben.

Auf den Inhalt des jüngeren Textes brauche ich hier selbstverständlich nicht einzugehen; er enthält Angaben über gewisse dem Pflanzen- und Thierreiche angehörige Heilmittel und dürfte derselbe durch das, was Herr Director Schmidt in dem erwähnten Programm nach meinen Angaben mittheilt, genügend charakterisirt sein. Was die äussere Erscheinung angeht, so möchte ich jetzt nach vielfältiger genauerer Prüfung indess noch nachtragen, dass nur auf der Seite des einen Blattes, auf der kein Bild angebracht ist, sich ein be- 
sonderes, mit scharfem Instrumente eingedrücktes, in zwei Verticalen eingeschlossenes, 20zeiliges Linienschema für den jüngeren Text befindet; auf den anderen Seiten hat man kein neues Schema angebracht, sondern sich nach dem in ähnlicher Weise angefertigten, aber 28 Zeilen in engeren Abständen zeigenden Liniensystem des älteren Textes gerichtet, ohne dasselbe jedoch unmittelbar zu benutzen; übrigens ist auf der vorerwähnten einen Seite die Schriftcolumne des neuen Textes um $21 \mathrm{~mm}$ breiter als die des älteren und steht bei einer Gesammtbreite des Blattes von $22,2 \mathrm{~cm}$ um $16 \mathrm{~mm}$ von dem jetzigen Rande zur Rechten ab; auf dem zweiten Blatte beträgt diese Entfernung für die beiden Texte $24 \mathrm{~mm}$. Der jüngere Text ist ferner mit einer gelbbraunen Tinte geschrieben, die sich für den Leser ganz gut von dem auf der einen Seite gelbgrau, auf der anderen Seite bräunlich-gelb gefärbten, starken und etwas spröden Pergament abhebt, aber der Deutlichkeit der photographischen Aufnahme nicht allzu förderlich war; die Schrift selbst ist, wie ich schon früher angab, eine kräftige, aber rohe Halbunciale; dieselbe zeigt zudem einmal die der eigentlichen Unciale angehörenden Charaktere in auffällig grosser Zahl, sodann kommen hie und da Formen vor, in denen man deutlich die Keime $z u$ der späteren karolingischen Minuskel erkennen kann; in der keulenförmigen Bildung der sogenannten Oberschäfte tritt eine gewisse Aehnlichkeit wiederum mit der bei Wattenbach-Zangemeister, Exempla codd. litteris maiusculis scriptorum, Taf. 44 abgebildeten Kölner Handschrift, die die Herausgeber dieser Sammlung eher noch dem ausgehenden 6 . als dem 7. Jahrhundert zuschreiben möchten, zu Tage. Für so alt glaube ich die spätere Schrift auf unseren Blättern indess doch nicht halten zu können; die Roheit der Uncialschrift, die wir in den Rubren der ziemlich zahlreichen kleineren Abschnitte treffen, und die barbarische Sprache, sowie vielfältig vorkommende Schreibfehler bestärken mich vielmehr darin, an der früher schon getroffenen Schätzung festzuhalten und als Entstehungszeit das spätere 7., vielleicht sogar den Anfang des 8. Jahrhunderts anzunehmen.

Muss hiernach schon der vorher auf diesen Blättern durch Radiren beseitigte Text ein erheblich höheres Alter besitzen, 
so findet das auch in den Formen der für ihn verwendeten Halb-Unciale, wie dieselben sich zuerst in den freien Räumen neben den Bildern des jüngeren Textes bemerkbar machten und durch Anwendung von Schwefel-Ammonium mit einiger Deutlichkeit hervortraten, seine Bestätigung; bis auf das a stimmt diese Schrift auffällig mit der anderer älterer und sicher zu datirender Proben überein, wie z. B. der 517 in Verona geschriebenen Handschrift der Vita b. Martini des Sulpicius Severus, von der sich bei Wattenbach-Zangemeister Taf̂. 32 eine Abbildung und im Nouveau traité de diplomatique III, pl. 46 eine Wiedergabe der Subscription findet. Die früher Herrn Director Schmidt gegenüber gemachte Angabe, dass auch hier Ueberschriften in reiner Uncialschrift angebracht gewesen seien, kann ich jetzt freilich nicht mit voller Sicherheit aufrecht erhalten; die einzige Ueberschrift, die ich bei näherer Prüfung nachweisen kann, ist derart mit späterer Schrift bedeckt, dass sich kein sicheres Urtheil darüber abgeben lässt, ob reine Unciale oder Halbunciale unter derselben liegt. Dass bei der Wiederbenutzung der Blätter zur Eintragung eines neuen Textes dieselben auf den Kopf gestellt worden sind, habe ich früher schon angeführt, dagegen nicht, dass man vielleicht gleichzeitig an den von Schrift freien Rändern der Blätter und zwar von dem einen oben und von dem anderen unten einen ungefähr $2 \mathrm{~cm}$ breiten Streifen abgeschnitten hat, denn die 28zeilige Columne steht nicht mehr in der Mitte der jetzt $28,2 \mathrm{~cm}$ hohen Blätter, sondern reicht auf dem einen nach oben $z u$, auf dem anderen nach unten hin näher an den jetzigen Rand heran; dies zugegeben, so müssen die Blätter ursprünglich im Verhältniss zu dem mit Schrift bedeckten Raume ein erheblich grosses Format besessen haben. An der Breite der Blätter ist, wie das schon oben angedeutet ist, nichts gekürzt worden; die Zahl der auf einer Zeile des Schemas stehenden Buchstaben ist natürlich schwankend; sie bewegt sich zwischen 32 und 40 und beträgt oft gerade das Mittel zwischen diesen beiden Zahlen; die Nummern der einzelnen Constitutionen ragen, obwohl sie meistens neben dem eingerückten und keine ganze Zeile füllenden Schlusse des Datums des voraufgehenden Abschnittes stehen, über das Colunnenschema auf den Rand heraus; auch 
die Anfangsbuchstaben des Textes, besonders das I des „Idem augustus" oder "Iidem augusti" steht mehrfach auf dem Rande.

Dank der gütigen Unterstützung, die mir Herr Geheimer Justizrath Prof. Fitting zu Theil werden liess, konnte ich schon bei meinen früheren Ausführungen mittheilen, dass die Blätter ursprünglich einer Handschrift des Codex Theodosianus angehört haben müssten; an der einen Stelle trat wenigstens der Anfang der c. 181. XII, 1 der Haenelschen Ausgabe, jedoch mit der Bezeichnung derselben als 182., deutlich bervor. Schwieriger war die Bestiminung des auf dem zweiten Blatte enthaltenen Stiickes, da dasselbe viel dichter von dem späteren Texte bedeckt war und an den wenigen von diesem freien Stellen nur gleichgültige Worte des älteren Textes lesbar wurden; erst ganz neuerdings glückte es mir in einer Zeile, wo ich den Anfang einer Constitution vermuthete, das Wort "Pacatianum" zu entziffern und festzustellen, dass Theile des IV. Titels des XIV. Buches hier vorlagen.

Ueber den Umfang der uns so überlieferten Stücke ergiebt sich daher Folgendes: Seite 1 des ersten Blattes beginnt mit den Worten ,societate coniunctos" des \& 1 der Const. 179, Lib. XII, Tit. I und reicht bis $\mathrm{zu}$,manus iniectione“ der Einleitung der Const. 181; die zweite Seite, auf der die Entfernung der ursprünglichen Schrift mit grösserem Erfolge als auf der ersten gegliickt ist, bringt die unmittelbare Fortsetzung und zwar allem Anscheine nach bis zu den Worten ,per ordinem propriae" der Const. 182; die letzte Zeile hier beginnt wenigstens, wie mit einiger Sicherheit erkennbar ist, mit , ,[uni]versis functionibus". Vom zweiten Blatte enthält die erste Seite zunächst den Schluss der 22. Constitution des III. Titels des XIV. Buches und zwar von ",[con]victus perpetuis" an, sodann die ganze Const. 1 des IV. Titels deutlich bis $\mathrm{zu}$,salutis etiam", während der Rest, obwohl hier noch ausreichend Platz zur Unterbringung desselben vorhanden gewesen wäre, erst auf der zweiten Seite erscheint; dann folgt auf letzterer beinahe die ganze 2. Const. des IV. Titels; da am Ende der 27. Zeile leidlich deutlich „daturi sunt" zu lesen ist, so kann die 28. und letzte Zeile nur mit den Worten ,in specie distra[henda"] geschlossen haben. 
Eine vollständige Collation dieser Theile mit der Haenelschen Ausgabe vorzunehmen, war ich leider nicht im Stande: vielfältig war die ältere Schrift durch Abwaschen und Radiren so gründlich getilgt, dass trotz mehrfacher. Anwendung der anderweit überaus wirksamen und sonst durchaus unschädlichen Tinctur keine lesbaren Spuren des ersten Textes zu Tage gefördert werden konnten; andererseits lagen oft die Zeilen beider Texte unmittelbar auf- und übereinander, sodass bei Anwendung eines Reagensmittels die spätere Schrift nur um so schwärzer hervorgetreten wäre und die jüngere nur desto gründlicher verdeckt hätte; stellenweis wäre ein solches Verfahren geradezu einer Vernichtung der Handschrift gleichgekommen; ganz fruchtlos ist indess auch die theilweise und beschränkte Vergleichung doch nicht gewesen; hie und da haben sich einige kleine Abweichungen ergeben, andererseits aber haben verschiedene Lesarten, die Haenel im Gegensatze zu einem Theile der bisherigen handschriftlichen Ueberlieferung wählte, hier ihre ausdrückliche Bestätigung gefunden. So steht in unserer Handschrift ebenso wie bei Haenel Sp. 1268 Z. 7 v. u. "singularum", Sp. 1269 Z. 1 v. u. ,soluto", Sp. 1384 Z. 4 v. 0 . ,edoctis"; dagegen hat statt des ,altioris curiae honores" Sp. 1270 Z. 4 v. o. die Handschrift ,altiores curiae honores", statt ,annumeret" Sp. 1385 Z. 4 v. o. die Handschrift ,adnumeret", statt ,pretium Sp. 1385 Z. 15 v. 0. die Handschrift ,praetium"; auch hat es den Anschein, als wenn in der Adresse der Const. 2, Lib. XIV, Tit. IV eine Abweichung bestünde; jedenfalls geht der Name des Beamten, an den dieselbe gerichtet ist, nicht, wie bei Haenel, mit „rino" (Verino) aus, sondern mit „,bino" oder „pino"; alles andere ist leider zu stark durch Rasur mitgenommen.

Ein ganz besonderes Interesse haben indess die vorliegenden Blätter noch dadurch, dass die oberen Theile derselben bereits einmal vor Anbringung der medicinischen Recepte rescribirt waren, und zwar sind auf Bl. 1 in gleicher Richtung mit der Schrift des Codex Theodosianus auf dem Rande zwei Zeilen angebracht und ist diese Eintragung alsdann bis zur 6. Zeile des Codex-Textes fortgesetzt, so dass im ganzen 8 Zeilen vorliegen; leider ruht hierbei die neue Schrift genau auf der alten, so dass die Entzifferung der ersten Rescription 
nur in geringem Umfange möglich ist; vor allem wird dieselbe aber noch dadurch erschwert, dass es eine sehr wenig starke Cursive ist, in der diese Eintragung angebracht wurde; eine Ausnahme hiervon macht nur die 1. Zeile, da dieselbe in Uncialschrift, wenn auch in sehr dünnleibiger, geschrieben ist; über dieselbe hin zieht sich in unmittelbarer Nälle des oberen Blattrandes noch eine mehrfach unterbrochene gerade Linie, die nur den Zweck einer Verzierung haben kann; jene oberste Zeile enthält wenigstens die deutlich lesbaren Worte: „De his qui de scientia dominorum", während von dem in der 2. Zeile sich unmittelbar hieran schliessenden Texte in Cursive nur , $\operatorname{cogn} \ldots .$. ceper" erkennbar ist; die 3. Zeile ist wiederum unleserlich bis auf ein "praef", das auf dem Rande les Blattes, soweit er von den beiden in Halbunciale geschriebenen Texten frei ist, steht; die. 4. Zeile, die nicht bis auf diesen Rand hinüberreicht, schliesst anscheinend mit „lib. ...t. X . . XXX; die 5., die wieder den Umfang der 3 . besitat, endet dagegen mit ,. . averit praefec . .", während die 6. sich nicht ganz so weit als die 4. erstreckt und am Ende ein „est ... lib. . . t. X . . . LXXI" - letztere Zahl freilich nicht mit voller Deutlichkeit - zeigt; in der 7. Zeile steht in der Mitte ziemlich leicht lesbar "abscentibus dominis" und der Schluss scheint mit dem der Zeile 5 bis auf das Fehlen des „ec" an ,praefec" übereinzustimmen; die 8. Zeile ist der 6. entsprechend gestaltet, doch dürfte hinter dem "est" noch ein ,praec" oder ,praet" stehen, wogegen aber die grössere Zahl am Schlusse fehlt. - Auf der zweiten Seite dieses Blattes ist von Cursivschrift durchaus nichts mehr bemerkbar, dagegen bringt Blatt 2 auf jeder seiner Seiten cursive. Eintragungen, aber auf dem Rande nur je eine Zeile, während das Weitere mit den beiden anderen Texten zusammenfällt; ausserdem sind unmittelbar am Rande der zweiten Seite eine Reihe nach oben geöffneter Bogen sichtbar, diese dürften aber wohl nur die untere Hälfte einer Verzierung sein, die ehemals aus zwei in sich verschlungenen Schlangenlinien bestand; die oben erwähnte Verstiimmelung der Blattränder muss daher wohl erst nach Anbringung der cursiven Notizen stattgefunden haben; auch auf Seite 1 des zweiten Blattes sind bei dieser Gelegenheit vielleicht die Ober- 
schäfte einzeIner Buchstaben ihres Kopfes beraubt worden. Glücklicherweise ist diese sowie die 2. Zeile von anderweiten Beschädigungen auffällig verschont geblieben, und es lässt sich hier der Satz: „... di de duobus noceat, ex duobus reis promettendi alius in diem $\mid \ldots \ldots$ obligari potest. Inst. lib. III. t. XYI. k. CCCLXXXIII." herauslesen. Um so schlimmer ist der Zustand der übrigen 3 Zeilen in Cursive; bis auf ein „st" auf dem Rande am Ende der 2. und bis auf Spuren einer grossen Zahl am Schlusse der 3. ist alles unleserlich; doch ist vielleicht hier nur der voraufgehende Satz wiederholt wordèn, denn auch in den beiden cursiven Zeilen der zweiten Seite, von denen die 2. auch schon mit der 1. Zeile des Codex Theodosianus zusammenfällt, taucht eine Bemerkung in ziemlich ähnlicher Fassung auf: de duobus . . . ceat, ex duobus reis promettendi in $\mathrm{e} . . \mathrm{d} \ldots$... ent . . . . . Inst. lib. III. t. XVI. k. CCCLXXXIII. In der That haben die so citirten Institutionenstellen eine ganz entsprechende Fassung, so dass sich die hier eingetragenen Notizen als Auszüge aus denselben bezeichnen lassen. Lag daher die Vermuthung nahe, dass auch die Eintragungen auf Blatt 1 derselben Quelle entstammen könnten, so ist es mir doch nicht gelungen daselbst etwas, was mit der Ueberschrift „De his qui de scientia dominorum" und den übrigen einzelnen Worten im Zusammenhange stehen könnte, nachzuweisen. Ich ziehte es daher vor, diese meine durch vielfältige, eingehende Prüfung der Blätter gewonnenen Beobachtungen in ihrem gegenwärtigen, fragmentarischen Zustande hier mitzutheilen und hoffe, dass es berufeneren Händen gelingen möchte, diese Anhaltspunkte wissenschaftlich erfolgreich weiter zu verwerthen.

Was schliesslich das Alter der Cursivschrift angeht, so möchte ich die vorliegenden Formen für etwas jünger halten als die in den Ravennatischen Urkunden der zweiten Hälfte des 6. Jahrhunderts vorkommenden (s. F. Massmann, Die gothischen Urkunden von Neapel und Arezzo, und Marini, I Papiri diplomatici), aber doch wieder für erheblich älter als die, die z. B. die langobardische Notariatsurkunde von $\mathbf{7 2 5}$ bei Fumagalli, Delle istituzioni diplomatiche vol. I tav. 2 zeigt; gerade an dem an unser ,promettendi" anklingenden ,promettit" der letzteren kann man recht deutlich den Alters- 

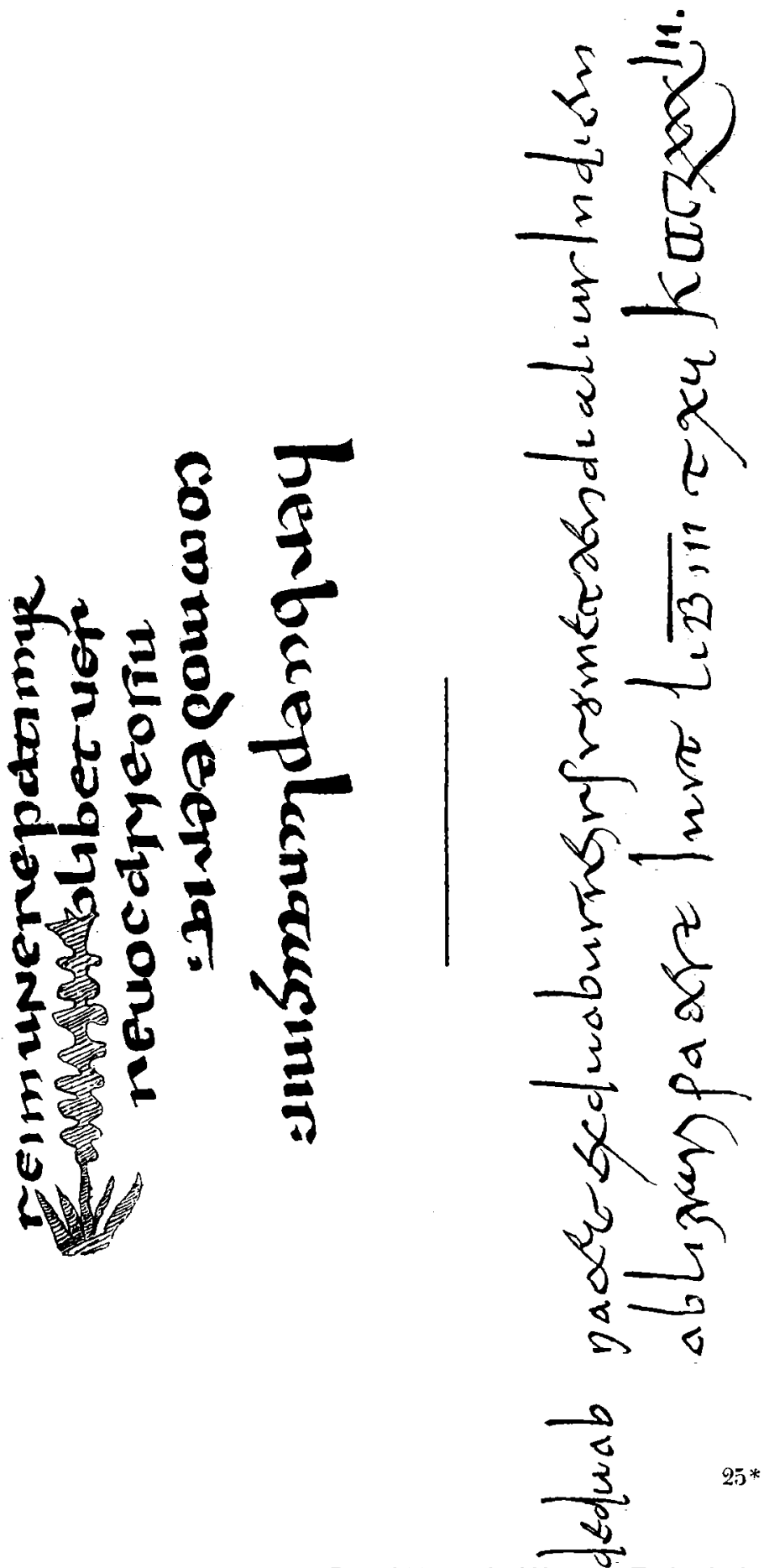
unterschied der Schrift erkennen. Die hier beigefügte $\mathbf{A b}$ bildung der einen in Cursive ausgeführten Bemerkung ist durch freihändige Nachzeichnung hergestetlt, da sich bei der blassen Farbe der Züge eine Pause nicht nehmen liess; die Proben der Uncialschrift sind mit Hülfe einer Durchzeichnung anmittelbar dem Originale entnommen; die schraffirte Figur stellt den Stachel eines Skorpions dar, der neben dem Recept gegen den Stich desselben im jüngeren Texte mit braunrother Farbe eingemalt ist.

Der wissenschaftliche Werth des interessanten Fundes, über welchen Herr Prof. Schum im vorstehenden berichtet, besteht für uns Juristen, wie mir scheint, nicht bloss darin, dass er zum Texte des Codex Theodosianus einige beachtenswerthe Varianten liefert, sondern und vor allen Dingen auch darin, dass er unsere Kenntniss der Geschichte des römischen Rechtes in mehrfacher Hinsicht bereichert. Denn erstens zeigen die Halberstädter Bruchstukcke in ihrer ersten Rescription: Beseitigung des Textes des Codex Theodosianus zu Gunsten juristischer Bemerkungen mit Institationencitaten schon um das Ende des 6. Jahrhunderts, ganz augenscheinlich, wie in Italien das vorjustinianische Recht, insbesondere der theodosische Codex, durch das Justinianische alsbald verdrängt wurde, - ein neuer Grund, Rechtsbüchern, welche, wie der Brachylogus, eine Benutzung des Breviars aufweisen, die italienische Herkunft abzusprechen. ${ }^{1}$ ) Sodann aber verdient auch die Form dieser Institutionencitate alle Beachtung. Es heisst zweimal übereinstimmend: Inst. lib. III. t. XVI. $k$. CCCLXXXIII. Es wird also der Bezeichnung der Stelle $(\S 2$ Inst. de duob. reis 3,16 ) nach Massgabe der von den Verfassern des Rechtsbuches selbst herrührenden Eintheilung beide Male in höchst merkwürdiger Weise noch eine Capitelzahl

1) Kein Widerspruch hiemit liegt in der Benutzung des echten Codex Theodosianus von Seite des Cardinals Deusdedit, welche ich selhst in dem folgenden Aufsatze (S. 379 ff.) nachweise, und in der Canonensammlung des Anselm von Lucca, für welche sie schon von Savigny nachgewiesen worden ist. Denn am Ende des 11. Jahrhunderts benutzten die Vorkämpfer der päpstlichen Partei sehr unbedenklich alles, was sie irgend zu Gunsten der Kirche, etwa in der reichen Bibliothek des Vaticans, auftreiben konnten. Vgl. S. 378 bei III, 12. 
beigesetzt. Spuren der Anführung von Institutionenstellen als capita lassen sich auch sonst nachweisen. So in einer der jüngeren Turiner Institutionenglossen: nr. 252 (10. Jahrhundert). S. Zeitschr. f. Rechtsgesch. VII, S. 67. Ferner in der Pistoieser Handschrift des Justinianischen Codex aus dem 10. und in der Pariser Handschrift desselben aus dem 11. Jahrhundert, hier wie dort in einer Bemerkung zu der L. $22 \mathrm{G}$. de furtis 6, 2. S. Codex Iustin. rec. P. Krueger p. XVIII. not. 5. Endlich in den Schriften des Cardinals Deusdedit aus dem Ende des 11. Jahrhunderts. S. unten S. $378 \mathrm{f}$. Allein hier sind durchweg mit den capita die 'Titel selbṣt gemeint, während in unserem Fall noch neben der Angabe des Titels die Verweisung auf ein Capitel auftritt. Auffallend und neu ist aucl die grosse Capitelzahl: 383. Man wird unwillkürllich auf die Vermuthung gebracht, dass schon in den Scbulen des 6. und 7. Jahrhunderts die Institutionen des bequemeren -Citirens halber, ähnlich der noch uns geläufigen Paragrapheneintheilung, in kurze Capitel eingetheilt, diese Capitel aber, so wie das ja schon damals auch mit denen des Julianischen Novellenauszuges geschah (s. Iuliani Epit. Novell. instr. G. Haenel p. XXXVIII.), fortlaufend gezählt worden seien (wobei nicht zu vergessen, dass die Institutionen und dieser Auszug die beiden am meisten gebrauchten Stïcke des Corpus iuris waren). Da nach sachlichen Rücksichten anzunehmen ist, dass die gedachte Capiteleintheilung mit der neueren Paragrapheneintheilung im ganzen übereingestimmt haben müsste, so babe ich, um einen etwas festeren Anhalt zu gewinnen, die Paragraphen bis zu $\S 2$ Inst. de duob. reis mit Einschluss derjenigen des Prooemium in der Krügerschen Ausgabe gezählt, dabei aber auch die principia als besondere Paragraphen mitgezählt. Das hat im ganzen $537 \mathrm{Abschnitte} \mathrm{ergeben.} \mathrm{Bedenkt} \mathrm{man,} \mathrm{dass} \mathrm{die} \mathrm{Zahl}$ der Paragraphen im Laufe der Zeit eine stetige Neigung zur Vermebrung gezeigt hat und noch in der Krügerschen Ausgabe diese Neigung zeigt, so wird man den Unterschied der beiden Zahlen 383 und 537 nicht gar zu gross finden und demnach obiger Vermutbung eine gewisse Wabrscheinlichkeit nicht absprechen können.

Fitting. 